\title{
LETTER
}

doi:10.1017/S1041610214000064

\section{Raising the bar: a comparative analysis of patients with Early Onset Alzheimer's disease}

Early onset Alzheimer's disease (EOAD) poses considerable challenges to physicians both in diagnostics and treatment, to patients and caregivers trying to cope with a debilitating illness at a young age and a healthcare system that is not geared to cater to degenerating illnesses striking young persons (Van Vliet et al., 2012). Routine procedures and screening measures for elderly people possibly stricken by dementia do not assess younger dementia patients in a favourable fashion. Physicians at an outpatient clinic diagnosing elderly patients, with well established standardized cognitive batteries for an older age norm, i.e. above 65 years may have unadjusted assumptions to account for in younger patients with symptoms of EOAD (Smits et al., 2012). Although a common battery of tests is internationally widely applied in the evaluation of cognitive impairment, these tests have not been validated extensively in a sample population of $\mathrm{AD}$ under the age of 65 .

In this letter, we compare briefly the clinical approach to patients with Early Onset Alzheimer's Disease (EOAD), based on the routines from two clinics located in Oslo, Norway and Boston, USA. The Norwegian Memory Clinic has from the time of referral to getting an appointment an average waiting period of 12 to 14 weeks. The criteria applied for ascertaining a diagnosis of EOAD, is based upon the guidelines specified in the ICD research criteria version 10 (WHO). To start with, the clinic specialists see both the patient and his/her informant for clinical history. Then, with the patient alone in the room, neuropsychological testing, and a complete general physical exam including somatic status are conducted. A neurological examination is not usually performed unless the patient demonstrates clear focal neurological manifestations. The first assessment often lasts for two until three hours. The bedside neuropsychological tests consist of a standardized battery. When no clear pattern of impairment is found corresponding to any known type of dementia or mild cognitive impairment (MCI) or when results are inconclusive, a referral for detailed and elaborate neuropsychological testing by a trained neuropsychologist is sought.

The clinic for Early Onset Dementia in Neuropsychiatry Unit at Massachusetts General
Hospital (MGH) in Boston, USA has a shorter time from a referral to getting an appointment with an average waiting period of 6 to 12 weeks (Chemali et al., 2012). The criteria for ascertaining a diagnosis of EOAD are the same as in Norway. The specialist will see both the patient and the informant to take a clinical history. Then, the patient is seen alone for the neurobehavioral testing and to complete a general physical and neurological exam. Commonly, the first assessment will last 90 minutes where a baseline neurological exam is mandatory. Similar to Norway, the patient's spouse, partner, offspring or next of kin is invited to a proxy interview. The first session with the informant/caregiver will take place without the patient in the room.

In Oslo, an average follow up consultation will last approximately 45 to 60 minutes. An EOAD patient will be scheduled for a follow up within a year of their first intake appointment. If medications have been prescribed (psychoactive, cholinesterase inhibitors or others) a routine ECG is performed and a closer follow up will occur within three to six months, or sooner if patient or caregiver report unwanted side effects or compliance issues. In a similar fashion to the Norwegian approach, on average, a follow up visit in Boston will last 60 minutes. EOAD patients are scheduled at MGH for follow up within three months of their first intake appointment. And comparable to Norway, an appointment is made sooner if medications have been prescribed or when the patient or caregiver relay unwanted side effects or compliance issues. The followup session in both clinics addresses the issues of safety at home and in the community, driving ability and the patient functioning and coping at work, the dynamics of the couple, how and when to involve the children and whether there are financial issues to consider. A social work assessment is considered vital at this stage. There are similar methods in both countries for scoring depressive states in EOAD patients. Depression is a serious afflicting comorbidity in EOAD (Rosness et al., 2010) and integrating a screening for depression in EOAD patients on the initial visit and follow-ups is key to a comprehensive evaluation and appropriate treatment. We advocate that frequent follow-ups are needed given that EOAD patients may compensate for their cognitive decline early on in the course of the disease (Fairjones et al., 2011). Scheduling follow-ups for the patients and caregivers as often as every three months may promote better continuity of care, especially when 
medication has been prescribed or when in need for additional psychosocial support.

Both countries have the same procedures and requirements for brain Magnetic Resonance Imaging (MRI) scans, although there is a shorter wait to have one performed in Boston. A difference was however seen in the use of Single Photon Emission Computed Tomography (SPECT) and Positron Emission Tomography (PET) scans in Oslo compared to Boston. This variation in practice is primarily based on economical reasons since PET is more costly than SPECT in Norway, and therefore performed more seldom. Furthermore, PET scan with Pittsburgh compound B (PiB) is not performed in Norway. In Boston, PET scan is a routine part of the EOAD work-up and PET with $\mathrm{PiB}$ is conducted in accordance with research protocol. SPECT is rarely requested in Boston. Known cerebrospinal fluid markers for dementia (beta amyloid, total tau, and phosphorylated tau) are routinely and similarly collected in both Oslo and Boston.

Given the comparative summary above, the authors would like to open a debate cautioning the international community that "one size does NOT fit all" and press towards working together to achieve a golden standard in ascertaining EOAD diagnosis worldwide. The accuracy of an early and correct diagnosis is key to better care for the patient and the system he/she lives in. We urge the international research communities to validate adaptive scales targeting specifically EOAD patients and their caregivers.

\section{Conflict of interest}

None.

\section{Description of author's role}

Tor Atle Rosness, conceived and designed the study and wrote the paper, Knut Engedal assisted in drafting and revising the paper critically, Zeina Chemali helped in the design of the study, writing and revising the paper with critical appraisal of important intellectual content. All authors approved of the final version of the paper.

\section{Acknowledgments}

We wish to thank the National Association for Public Health and the Norwegian Centre for Dementia Research, for grants given (no grant number was issued). The authors would also like to thank the staff at the Neuropsychiatry Clinics, Massachusetts General Hospital, Boston, USA and remain grateful to all the services offered at the time of writing the paper.

\section{References}

Chemali, Z., Schamber, S., Tarbi, E., Acar, D. and Avila-Urizar, M. (2012). Diagnosing early onset dementia and then what? A frustrating system of aftercare resources. Internal fournal of General Medicine, 5, 81-86.

Fairjones, S. E., Vuletich, E. J., Pestell, C. and Panegyres, P. K. (2011). Exploring the role of cognitive reserve in early-onset dementia. American fournal of Alzheimers Disease and Other Dementias, 26, 139-144.

Rosness, T. A., Barca, M. L. and Engedal, K. (2010). Occurrence of depression and its correlates in early onset dementia patients. International Fournal of Geriatric Psychiatry, 25, 704-711.

Smits, L. et al. (2012). Early onset Alzheimer's disease is associated with a distinct neuropsychological profile. fournal of Alzheimers Disease, 30, 101-108.

Van Vliet, D. et al. (2012). Time to diagnosis in young-onset dementia as compared with late-onset dementia. Psychological Medicine, 43, 423-432.

Tor Atle Rosness, ${ }^{1} \mathrm{KNUT}_{\text {ENGedal }}{ }^{2}$ AND ZEINA CHEMALI ${ }^{3}$

${ }^{1}$ Institute of Health and Society, University of Oslo, Norway

${ }^{2}$ Norwegian Centre for Dementia Research, Department of Geriatric Medicine, Ullevaal University Hospital, Oslo, Norway

${ }^{3}$ Neuropsychiatry Clinics, Departments of Neurology and Psychiatry, Massachusetts General Hospital, Harvard Medical School, Boston, USA, Email: t.a.rosness@medisin.uio.no 